\title{
Termitofauna (Insecta, Isoptera) em Remanescentes de Floresta Estacional Semidecidual em São Leopoldo, Rio Grande do Sul, Brasil
}

\author{
Daniela Faria Florencio ${ }^{1} \&$ Elena Diehl ${ }^{1,2}$
}

${ }^{1}$ PPG em Biologia: Diversidade e Manejo de Vida Silvestre; Laboratório de Insetos Sociais, UNISINOS, Caixa Postal 275, $93001-970$

São Leopoldo-RS.

${ }^{2}$ Pesquisadora do CNPq. elena.diehl@ pesquisador.cnpq.br

\begin{abstract}
Assemblages of termites (Insecta, Isoptera) in fragments of stationary semideciduous forest in São Leopoldo, Rio Grande do Sul State, Brazil. It has been reported that forest fragmentation modifies the richness and/or the abundance of communities. To test this hypothesis it was evaluated the fauna of termites of six fragments of stationary semideciduous forest and an area in early succession. In each one it was designed a transect (100 m x $2 \mathrm{~m}$ ), divided into 20 plots (5 m x $2 \mathrm{~m}$ ). In ten of these plots the collections were conducted under stones, in the nests, trunks and branches, over/under trunk barks and in five soil samples $\left(340 \mathrm{~cm}^{3}\right)$ of the A-horizon by plot with a sampling effort of 60 minutes/plot. The composition, the observed and estimated species richness, the relation between area and observed richness, as well as the occurrence and dominance patterns of each species were analyzed. Eleven species from nine genera and two families (Kalotermitidae and Termitidae) were collected, rising the number of species registered in the state from 14 to 18 . The subfamily, Apicotermitinae with the upper species richness $(S=6)$, was found in five of the seven areas, while Nasutitermitinae, with the second highest number of species $\left(\mathrm{S}_{\mathrm{obs}}=4\right)$, was collected in all areas. The richness varied from two to six species, and was not related to the area size. Five species were considered rare, but the others were intermediate. The areas were grouped in four groups with $60,57,50$ and $40 \%$ of similarity, with the species composition as the highest difference between them, suggesting that fragmentation of the local forest influenced more the composition than the richness of termites.
\end{abstract}

KEYWORDS. Kalotermitidae; Neotropical; Termitidae.

RESUMO. Termitofauna (Insecta, Isoptera) em remanescentes de floresta estacional semidecidual em São Leopoldo, Rio Grande do Sul, Brasil. Tem sido relatado que o desmatamento e a fragmentação florestal alteram a riqueza e/ou a abundância das comunidades animais, havendo uma relação positiva com o tamanho do fragmento. Visando testar esta hipótese, foram comparadas as termitofaunas de seis remanescentes de floresta estacional semidecidual e de uma área desmatada em início de sucessão vegetal, com diferentes tamanhos. Em dez setores intercalados de um transecto traçado em cada ambiente, os térmitas foram procurados sob pedras, no interior de ninhos, troncos e galhos, sobre/sob a casca dos troncos e em amostras de solo do horizonte-A. Por área, foram analisadas: a composição da termitofauna, as riquezas de espécies observada e estimada e a relação área-riqueza observada. No total, foram coletadas 11 espécies de nove gêneros e duas famílias (Kalotermitidae e Termitidae). De Termitidae, a subfamília Apicotermitinae apresentou a maior riqueza de espécies $\left(\mathrm{S}_{\mathrm{obb}}=6\right)$ e foi encontrada em cinco áreas, enquanto Nasutitermitinae, com a segunda maior riqueza $\left(S_{\mathrm{obs}}=4\right)$, foi registrada nos sete ambientes. A riqueza variou de duas a seis espécies, mas não mostrou relação com o tamanho da área. A análise de agrupamento revelou a existência de quatro grupos de áreas que pela composição de espécies apresentaram similaridades variando de 60 a $40 \%$. Pelos padrões de ocorrência, cinco espécies (uma/ambiente) foram categorizadas como raras e as demais como intermediárias. Os resultados sugerem que a fragmentação e o desmatamento da floresta estacional semidecidual local interferiram mais na composição do que na riqueza de espécies de térmitas.

PALAVRAS-CHAVE. Kalotermitidae; Neotropical; Termitidae.

Os térmitas ocorrem nas áreas tropicais e temperadas, entre os paralelos $45^{\circ}-48^{\circ} \mathrm{N}$ e $45^{\circ} \mathrm{S}$ (Wood 1975). Existem aproximadamente 2.600 espécies e 281 gêneros descritos, sendo que a região Neotropical, com 481 espécies, possui a terceira maior riqueza (Kambhampati \& Eggleton 2000). Para o Brasil, ainda há discrepâncias quanto à riqueza, estando registradas em torno de 250 espécies (Fontes \& Araujo 1999), ou 280 (Cancello \& Schlemmermeyer 1999), ou ainda de cerca de 290 (Constantino 1999). Porém, os autores concordam que certamente estes números devem se elevar com a ampliação dos estudos termitofaunísticos no país. Para o Rio Grande do Sul, os dados de catálogos (Araujo 1977; Constantino 1998; Fontes 1998a) complementados com trabalhos recentes (Castro
\& Diehl 2003; Diehl et al. 2005) registram apenas 14 espécies. Esta reduzida riqueza pode ser decorrente tanto das características climáticas da região Subtropical (Eggleton et al.1994; Diehl et al. 2005), como da falta de taxonomistas e estudos locais (Diehl-Fleig et al. 1995).

A riqueza local de espécies está relacionada com características ambientais como altitude, temperatura, pluviosidade, tipo e estrutura da vegetação, de modo que a frequiência de ocorrência dos térmitas reflete a disponibilidade de recursos e suas relações intra- e interespecíficas (Eggleton 2000). Assim como em outros ambientes, nas florestas tropicais a termitofauna é suscetível às alterações dos hábitats (Eggleton et al. 1995; Jones \& Eggleton 2000), sendo que o desmatamento 
e a fragmentação florestal interferem nos grupos funcionais, reduzindo a diversidade de espécies (De Souza \& Brown 1994; Bandeira \& Vasconcelos 2002). A fragmentação das florestas leva à redução de área, isolamento e modificação na estrutura da vegetação, bem como à eliminação de habitats causando a perda de indivíduos e mesmo de populações inteiras. Entretanto, o número de espécies pode aumentar, diminuir ou mesmo se manter constante, o que não significa que a fauna não sofreu com a fragmentação (De Souza et al. 2001).

O município de São Leopoldo faz parte da região da floresta estacional semidecidual classificada pelas características fisionômicas e ecológicas como floresta de terras baixas. Com o início do processo de urbanização, a floresta foi fragmentada dando espaço à agricultura, pecuária e expansão das zonas urbana e industrial, além de muitas áreas terem sido drenadas ou aterradas (Teixeira et al. 1986). Partindo da hipótese que essa fragmentação teria levado algumas espécies à extinção local, enquanto outras teriam sido introduzidas, mantendo assim um equilíbrio da riqueza, seria esperado que as atuais termitofaunas nos remanescentes florestais apresentassem riqueza de espécies semelhante, mas distintas composições. Para avaliar essa hipótese comparou-se as comunidades de térmitas quanto ao número e composição de espécies, padrão de ocorrência e de dominância de cada uma em seis remanescentes florestais e em uma área desmatada mas já em processo de sucessão vegetal, além de ter sido avaliado se a riqueza de espécies apresentaria correlação com o tamanho da área.

\section{MATERIALE MÉTODOS}

Áreas de Estudo. O município de São Leopoldo está localizado na região da Encosta Inferior do Nordeste do Rio Grande do Sul, 26 metros acima do nível do mar. Segundo a classificação de Köeppen, a região apresenta clima mesotérmico subtropical, com períodos temperados. A temperatura média anual é de $19,7^{\circ} \mathrm{C}$ (máxima $40,4^{\circ} \mathrm{C}$ e mínima $\left.0,6^{\circ} \mathrm{C}\right), 76 \%$ de umidade relativa média do ar e precipitação pluviométrica de $1.390 \mathrm{~mm}$ por ano (Hackbart 2004).

As coletas dos térmitas foram feitas em seis remanescentes florestais e em uma área desmatada em início de sucessão vegetal, afastadas entre si de um a $15 \mathrm{~km}$. Algumas características das áreas são apresentadas na Tabela I.

Coletas. De novembro de 2003 a junho de 2004, em cada área foi traçado um transecto ( $100 \mathrm{~m} \times 2 \mathrm{~m}$ ), dividido em 20 setores de $10 \mathrm{~m}^{2}(5 \mathrm{~m} \times 2 \mathrm{~m})$. Em dez setores intercalados e em zig-zag, os térmitas foram procurados na superfície do solo, na serrapilheira, na base das árvores, sob pedras, troncos e galhos, no interior de ninhos arborícolas e epígeos, troncos em decomposição, sobre/sob a casca dos troncos, com esforço amostral de 30 minutos/setor (adaptado de Eggleton et al. 1995; Davies 1997; Jones \& Eggleton 2000). Paralelamente, também por setor, foram retiradas cinco amostras $\left(340 \mathrm{~cm}^{3}\right)$ de solo do horizonte-A, acondicionadas em sacos plásticos individuais e triadas, em laboratório, por 30 minutos. Assim, para cada setor foi utilizado um esforço de amostragem total de uma hora.

Identificação do Material. As amostras foram triadas e os térmitas transferidos para vidros âmbar com álcool $80 \%$. Para a identificação em nível de gênero, foram adotadas as chaves dicotômicas de Fontes $(1985,1992)$ e de Constantino (1999). Os espécimes foram separados em morfo-espécies e parte do material foi enviado ao Laboratório de Termitologia do Departamento de Zoologia da UnB, para identificação em nível de espécie. O material testemunho está depositado na Coleção de Isoptera do Laboratório de Insetos Sociais da Universidade do Vale do Rio dos Sinos - UNISINOS, São Leopoldo (RS).

Análises Faunísticas. Além da determinação da composição e riqueza de espécies observada $\left(\mathrm{S}_{\mathrm{obs}}\right)$ foram realizadas análises faunísticas da termitofauna de cada local, visando avaliar:

Tabela I. Características das áreas onde foram avaliadas as comunidades de térmitas no município de São Leopoldo, Rio Grande do Sul, Brasil (nov/ 03 - jun 04).

\begin{tabular}{|c|c|c|c|}
\hline Área & Coordenadas Geográficas & Tamanho(ha) & Vegetação \\
\hline A - Área de Preservação & $29^{\circ} 47^{\prime} 31,8^{\prime \prime} \mathrm{S}-51^{\circ} 09^{\prime} 07,3^{\prime \prime} \mathrm{W}$ & 1,18 & $\begin{array}{l}\text { remanescente florestal com sub-bosque; vegetação rasteira } \\
\text { densa; muita serrapilheira; solo bem drenado }\end{array}$ \\
\hline B - Base Ecológica Rio Velho & $29^{\circ} 46^{\prime} 01,9^{\prime \prime} \mathrm{S}-51^{\circ} 10^{\prime} 54,2^{\prime \prime} \mathrm{W}$ & 8,52 & $\begin{array}{l}\text { remanescente de floresta inundável às margens de meandro } \\
\text { de rio; sem sub-bosque; solo desnudo compactado e com } \\
\text { intenso ressecamento nos períodos sem lâmina d'água }\end{array}$ \\
\hline C - São José & $29^{\circ} 46^{\prime} 0,8^{\prime \prime} \mathrm{S}-51^{\circ} 08^{\prime} 20,1^{\prime \prime} \mathrm{W}$ & 1,04 & $\begin{array}{l}\text { remanescente florestal sem sub-bosque; pouca vegetação } \\
\text { rasteira; muita serrapilheira; solo muito compactado }\end{array}$ \\
\hline D - Cristo Rei & $29^{\circ} 47^{\prime} 14,8^{\prime \prime} \mathrm{S}-51^{\circ} 09^{\prime} 07,8^{\prime \prime} \mathrm{W}$ & 0,98 & $\begin{array}{l}\text { remanescente florestal com dossel fechado; muita } \\
\text { serrapilheira; solo úmido }\end{array}$ \\
\hline E - Morro do Espelho & $29^{\circ} 46^{\prime} 25,8^{\prime \prime} \mathrm{S}-5^{\circ} 08^{\prime} 20,0^{\prime \prime} \mathrm{W}$ & 0,53 & $\begin{array}{l}\text { remanescente florestal denso com sub-bosque; pouca } \\
\text { serrapilheira; solo bem drenado }\end{array}$ \\
\hline F - Campestre & $29^{\circ} 47^{\prime} 03,4^{\prime \prime} \mathrm{S}-5^{\circ} 05^{\prime} 16,6^{\prime \prime} \mathrm{W}$ & 3,00 & $\begin{array}{l}\text { remanescente florestal heterogêneo com e sem sub-bosque; } \\
\text { solo desnudo e úmido }\end{array}$ \\
\hline G - Imperatriz Leopoldina & $29^{\circ} 45^{\prime} 46,7^{\prime \prime} \mathrm{S}-51^{\circ} 07^{\prime} 59,3^{\prime \prime} \mathrm{W}$ & 0,50 & $\begin{array}{l}\text { início de sucessão vegetal; rara vegetação arbustiva; solo } \\
\text { muito úmido }\end{array}$ \\
\hline
\end{tabular}


Riqueza estimada $\left(\mathrm{S}_{\text {est }}\right)$, pelo estimador jackknife de primeira ordem do programa EstimateS versão 7 (Colwell 2004).

Similaridade, com análise de agrupamento pela medida do coeficiente de distância de Sörensen (baseado na presença/ ausência das espécies) entre os ambientes, utilizando o programa Krebs/WIN versão 0.9 (Krebs 1997).

Relação área $\mathbf{x} \mathbf{S}_{\text {obs, }}$, por análise de regressão linear pelo programa SYSTAT 10.0 (Wilkinson 2000) relacionando a riqueza de espécies observada com o tamanho da área transformado em logaritmo na base 10 de cada ambiente.

Padrão de ocorrência, número de setores em que a espécie foi encontrada, dividido pelo número total de setores avaliados x 100. As espécies foram categorizadas de acordo com o seu padrão de ocorrência em: rara, quando presente em 1 a $10 \%$ dos setores; esporádica, em 11 a 40\%; comum: em 41 a $70 \%$; freqüente, em 71 a $99 \%$, e constante quando presente em $100 \%$ dos setores avaliados.

Padrão de dominância, número de ocorrências de cada espécie dividido pelo número total de ocorrências de térmitas por local x 100. Quanto ao padrão de dominância, a espécie foi categorizada como rara, quando a ocorrência variou de 1 a $10 \%$ do total; acessória, de 11 e 49\%; e dominante, quando a ocorrência da espécie variou de 50 a $100 \%$ do total de ocorrências de térmitas por local.

A combinação dos padrões de ocorrência e de dominância por ambiente foi utilizada como uma indicação da abundância de cada espécie. Assim, uma espécie foi considerada comum, quando constante e dominante em um ambiente; intermediária, quando a) rara, esporádica, comum ou freqüente e acessória; b) esporádica, comum, freqüente ou constante e rara; e, finalmente, c) rara, no caso de ter padrão de ocorrência raro e dominância também rara (adaptado de Scatolini \& PenteadoDias 2003).

\section{RESULTADOS}

No total dos seis remanescentes florestais e na área em sucessão vegetal foram coletadas 11 espécies (Tabela II) pertencentes a nove gêneros e duas famílias (Kalotermitidae e Termitidae). Duas amostras não puderam ser identificadas em nível de gênero, uma das quais de Apicotermitinae (Termitidae) e uma de Kalotermitidae contendo apenas indivíduos imaturos o que impossibilita a identificação.

Por local, a riqueza variou de duas a seis espécies, com a subfamília Apicotermitinae apresentando a maior riqueza específica $(S=6)$ e ocorrendo em cinco dos sete ambientes avaliados (Tabela II). Os gêneros Grigiotermes (Apicotermitinae) e Nasutitermes (Nasutitermitinae) apresentaram a maior riqueza específica, cada um com duas espécies, enquanto os demais estiveram representados por uma única espécie. Indivíduos de Grigiotermes sp.1 foram coletados em cinco ambientes; de Nasutitermes jaraguae (Holmgren, 1910) em quatro; de Nasutitermes aquilinus (Holmgren, 1910) e Anoplotermes sp.1 em três. Paralelamente, indivíduos de Aparatermes sp.1, Ruptitermes sp.1, Cortaritermes fulviceps (Silvestri, 1901), Neocapritermes sp.1 e Rugitermes sp.1 foram encontrados em apenas um dos sete ambientes (Tabela II).

A maioria das 13 espécies coletadas foi categorizada com relação ao padrão de ocorrência e ao padrão de dominância como intermediária em quase todos os ambientes amostrados (Tabela II). No entanto, Grigiotermes bequaerti (Snyder \& Emerson, 1949), Grigiotermes sp.1, Neocapritermes sp.1, o apicotermitíneo e o calotermitídeo não identificados, foram categorizados como raros em cinco ambientes (Tabela II).

As curvas de riqueza estimada (Fig. 1) mostram que nos sete remanescentes o número de setores amostrados e o esforço amostral utilizado foram suficientes para detectar as espécies presentes. As maiores riquezas, observada e estimada (Tabela III), foram encontradas no remanescente do Campestre, seguidas pela Área de Preservação, Morro do Espelho e Imperatriz Leopoldina, enquanto as menores ocorreram na Base Ecológica, Cristo Rei e São José. Para todos os ambientes, a relação entre o tamanho da área e a riqueza de espécies observada não foi significativa $(\mathrm{F}=0,424 ; \mathrm{df}=1,5 ; \mathrm{P}=0,544)$.

A análise de agrupamento, utilizando a riqueza e a composição de espécies, permitiu reunir os sete ambientes em quatro grupos (Fig. 2). Os remanescentes florestais da Área de Preservação e do Morro do Espelho tiveram a mesma riqueza de espécies $(\mathrm{S}=5)$, das quais três em comum (Anoplotermes sp.1, Grigiotermes sp.1 e $N$. jaraguae), tendo sido reunidos em um grupo com $60 \%$ de similaridade. O agrupamento São José e Cristo Rei ficou muito próximo ao dos remanescentes anteriores $(57 \%)$, tendo mostrado menor riqueza $(\mathrm{S}=2), \operatorname{com} N$. jaraguae sendo comum à ambos. Em seguida, ficou o remanescente florestal da Base Ecológica Rio Velho (50\%) com duas espécies, das quais $N$. aquilinus foi comum à Área de Preservação e São José.

Os ambientes Campestre e Imperatriz Leopoldina (Fig. 2), com $55 \%$ de similaridade entre si, ficaram separados dos outros grupos, mostrando apenas $40 \%$ de similaridade com eles. As riquezas observadas foram, respectivamente, de seis e cinco

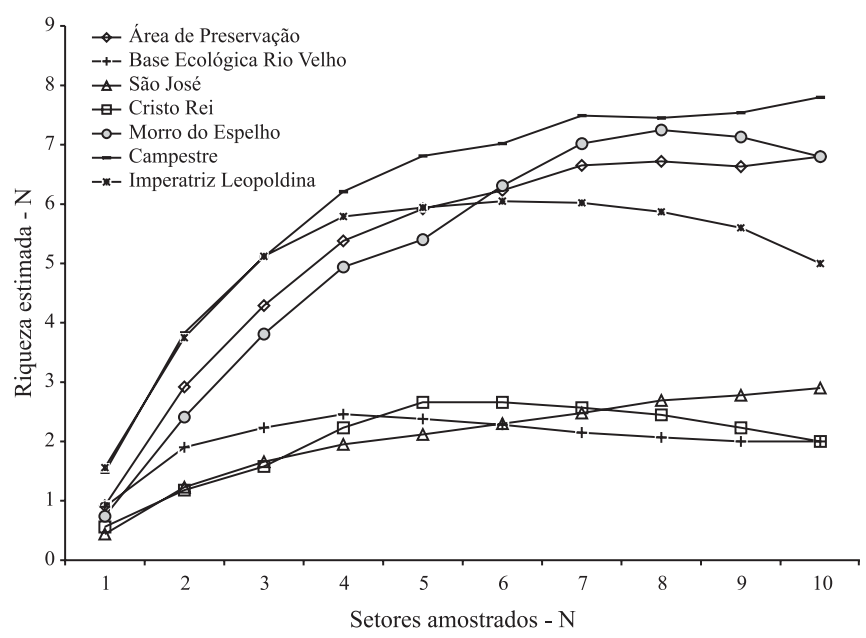

Fig. 1. Curvas de riqueza estimada (jackniffe de primeira ordem) das espécies de térmitas em seis remanescentes florestais e em uma área em sucessão vegetal. 
Tabela II. Número de setores $(\mathrm{N})$ em que a espécie foi coletada, freqüências (\%) dos padrões de ocorrência (PO) e de dominância (PD) e categorias (C) das espécies de térmitas em seis remanescentes florestais e em uma área em sucessão vegetal.

\begin{tabular}{|c|c|c|c|c|c|c|c|c|c|c|c|c|c|c|c|c|c|c|c|c|c|}
\hline \multirow{3}{*}{$\begin{array}{l}\text { Família / Subfamília } \\
\text { Espécie }\end{array}$} & \multicolumn{21}{|c|}{ Ambientes } \\
\hline & \multicolumn{2}{|c|}{$\mathrm{A}$} & \multicolumn{4}{|c|}{$\mathrm{B}$} & \multicolumn{4}{|c|}{$\mathrm{C}$} & \multicolumn{3}{|c|}{$\mathrm{D}$} & \multicolumn{3}{|c|}{$\mathrm{E}$} & \multicolumn{2}{|l|}{$\mathrm{F}$} & \multicolumn{3}{|c|}{ G } \\
\hline & $\mathrm{N} \mathrm{PO}$ & PD C & & N PO & $\mathrm{PD}$ & $\mathrm{C}$ & $\mathrm{N}$ & $\mathrm{PO}$ & $\mathrm{PD}$ & $\mathrm{C}$ & $\mathrm{N} P O$ & $\mathrm{PD}$ & $\mathrm{C}$ & N PO & $\mathrm{PD}$ & & $\mathrm{N} P O$ & $\mathrm{PD} \mathrm{C}$ & $\mathrm{N} \mathrm{PO}$ & PD & $\mathrm{C}$ \\
\hline \multicolumn{22}{|c|}{ 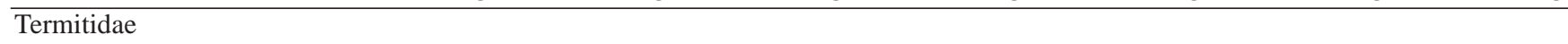 } \\
\hline \multicolumn{22}{|l|}{ Apicotermitinae } \\
\hline Anoplotermes sp.1 & 330,0 & $30,0 \mathrm{I}$ & & $-\quad-$ & - & - & - & - & - & - & - & - & - & 110,0 & 12,5 & & 315,0 & $18,8 \mathrm{I}$ & $-\quad-$ & - & - \\
\hline Aparatermes sp.1 & $-\quad-$ & $-\quad-$ & & - & - & - & - & - & - & - & - & - & - & - & - & - & - & $-\quad-$ & 420,0 & 26,7 & $7 \mathrm{I}$ \\
\hline Grigiotermes bequaerti & - & - - & & - & - & - & - & - & - & - & - & - & - & $-\quad-$ & - & - & 15,0 & $6,3 \mathrm{R}$ & 210,0 & 13,3 & $3 \mathrm{I}$ \\
\hline Grigiotermes sp.1 & 110,0 & $10,0 \mathrm{R}$ & & - & - & - & - & - & - & - & 220,0 & 40,0 & I & 220,0 & 25,0 & & 525,03 & $31,3 \mathrm{I}$ & 630,0 & 40,0 & \\
\hline Ruptitermes sp.1 & $-\quad-$ & $-\quad-$ & & - & - & - & - & - & - & - & - & - & - & 220,0 & 25,0 & & $-\quad-$ & $-\quad-$ & $-\quad-$ & - & - \\
\hline Gênero não identificado & $-\quad-$ & $-\quad-$ & & $-\quad-$ & - & - & - & - & - & - & - & - & - & 110,0 & 12,5 & & - & $-\quad-$ & 15,0 & 6,7 & $\mathrm{R}$ \\
\hline \multicolumn{22}{|l|}{ Nasutitermitinae } \\
\hline Cornitermes cumulans & - & - & & - & - & - & - & - & - & - & - & - & - & - & - & - & 315,01 & $18,8 \mathrm{I}$ & 210,0 & 13,3 & $3 \mathrm{I}$ \\
\hline Cortaritermes fulviceps & $-\quad-$ & - - & & $-\quad-$ & - & - & - & - & - & - & - & - & - & $-\quad-$ & - & - & 315,01 & $18,8 \mathrm{I}$ & $-\quad-$ & - & - \\
\hline Nasutitermes aquilinus & 220,0 & $20,0 \mathrm{I}$ & & 550,0 & 62,5 & I & & 10,0 & 20,0 & & - & - & - & $-\quad-$ & - & - & $-\quad-$ & $-\quad-$ & $-\quad-$ & - & - \\
\hline Nasutitermes jaraguae & 330,0 & $30,0 \mathrm{I}$ & & - & - & - & & 40,0 & 80,0 & & 330,0 & 60,0 & & 220,0 & 25,0 & & - & $-\quad-$ & - & - & - \\
\hline \multicolumn{22}{|l|}{ Termitinae } \\
\hline Neocapritermes sp.1 & - & - & & - & - & - & - & - & - & - & - & - & - & - & - & - & 15,0 & $6,3 \mathrm{R}$ & - & - & - \\
\hline \multicolumn{22}{|l|}{ Kalotermitidae } \\
\hline Rugitermes sp.1 & - & - & & 330,0 & 37,5 & I & - & - & - & - & - & - & - & - & - & - & $-\quad-$ & $-\quad-$ & - & - & - \\
\hline Gênero não identificado & 110,0 & $10,0 \mathrm{R}$ & & $-\quad-$ & - & - & - & - & - & - & - & - & - & $-\quad-$ & - & - & $-\quad-$ & $-\quad-$ & $-\quad-$ & - & - \\
\hline Riqueza & & 5 & & & 2 & & & & 2 & & & 2 & & & 5 & & 6 & & . & 5 & \\
\hline
\end{tabular}

$\mathrm{A}=$ Área de Preservação, B=Base Ecológica Rio Velho, C=São José, D=Cristo Rei, E=Morro do Espelho, F=Campestre, G=Imperatriz Leopoldina; $\mathrm{I}=$ intermediária, $\mathrm{R}=$ rara).

espécies, das quais três foram comuns a ambos: G. bequaerti, Grigiotermes sp.1 e Cornitermes cumulans (Kollar, 1832).

\section{DISCUSSÃO}

Para o Rio Grande do Sul estavam registradas 12 espécies de térmitas (Araujo 1977; Constantino 1998; Fontes 1998a). Mais recentemente, analisando apenas a termitofauna de ninhos epígeos, foram relatados pela primeira vez para o estado representantes do gênero Aparatermes (Castro \& Diehl 2003), além de uma espécie do gênero Dihoplotermes em um ninho epígeo de C. cumulans (Diehl et al. 2005). No presente estudo, outras cinco espécies (G. bequaerti, N. jaraguae, Grigiotermes sp.1, Rugitermes sp.1 e Ruptitermes sp.1) são registros novos, o que eleva para 18 o número de espécies no estado. No entanto, certamente esta riqueza é uma subestimativa decorrente da falta de levantamentos extensivos, além das dificuldades taxonômicas inerentes ao reduzido número de taxonomistas locais e no Brasil (Diehl-Fleig et al. 1995).

As curvas de riqueza estimada sugerem que o número de setores avaliados e o esforço amostral utilizado foi suficiente para amostrar todas ou quase todas as espécies presentes. No remanescente da Campestre e na área em sucessão vegetal Imperatriz Leopoldina foram encontradas as maiores riquezas de espécies, embora as amostras tenham sido obtidas, quase que somente, em ninhos epígeos. De acordo com Bignell \& Eggleton (2000), amostragens realizadas apenas em ninhos epígeos e/ou arborícolas podem subestimar a riqueza de espécies, pois a maioria vive em ninhos subterrâneos ou em troncos em decomposição. Nos demais ambientes, com mesmo protocolo de coleta e mesmo esforço amostral, a riqueza foi praticamente igual. Assim, não pode ser descartada a possibilidade do número de espécies aumentar, se forem feitas mais amostragens subterrâneas e na superfície do solo.

Apesar do pequeno número de espécies encontradas nos diversos remanescentes florestais e na área em sucessão, ele está de acordo com a riqueza esperada para regiões de clima subtropical a temperado (Eggleton 1994, 2000). Utilizando mesmo protocolo, Diehl et al. (2005) encontraram um total de oito espécies de térmitas na Floresta Nacional de São Francisco de Paula, caracterizada como floresta ombrófila mista, no nordeste do Rio Grande do Sul. Nos diversos fragmentos florestais amostrados a riqueza observada variou de duas a quatro espécies, sendo que a maior diferença entre eles residiu na composição.

A subfamília Apicotermitinae (Termitidae) além de apresentar a maior riqueza de espécies, ocorreu em maior número de ambientes. Os dois fragmentos (Base Ecológica Rio Velho e São José) onde não foram coletados apicotermitíneos, foram aqueles em que o solo é mais compactado, o que de acordo com Davies (2002) dificulta ou mesmo impede a sobrevivência destes térmitas. Segundo Mathews (1977), a ocorrência dos representantes dessa subfamília, possivelmente, está relacionada com a estrutura do solo, uma vez que maioria das espécies nidifica e/ou depende dele para alimentação. Destaca-se ainda que o remanescente florestal da Base Ecológica Rio Velho, por estar situado ás margens de um meandro antigo de rio, permanece inundado por longos períodos durante o ano. Já havia sido referido por Mill (1982) e Constantino (1992) menor riqueza de 
espécies em igapós e florestas inundáveis do que em florestas de terra firme na Amazônia. De acordo com Bignell \& Eggleton (2000), apesar de algumas espécies possuírem estratégias adaptativas, tais como construção de ninhos arborícolas, os térmitas são fortemente afetados quando seus habitats sofrem inundação.

Os táxons Aparatermes sp.1 e Ruptitermes sp.1(Apicotermitinae), C. fulviceps (Nasutitermitinae) e Neocapritermes sp.1 (Termitinae), todos da família Termitidae, foram encontrados em apenas um dos sete ambientes. Os representantes dos gêneros Aparatermes, Cortaritermes, Neocapritermes e Ruptitermes vivem em ninhos epígeos e/ou subterrâneos e são essencialmente geófagos. De acordo com Brauman et al. (2000), por definição, são geófagos os térmitas que consomem material altamente heterogêneo, incluindo desde matéria orgânica, tecidos vegetais fragmentados, paredes celulares lignificadas, xilema velho e seus subprodutos, até itens de alto valor nutricional como hifas e esporos de fungos, algas, pequenas raízes e pêlos radiculares.

Paralelamente, também foram coletados em apenas um ambiente (Base Ecológica Rio Velho), indivíduos de Rugitermes sp.1 (Kalotermitidae). Os representantes de Rugitermes, assim como os demais calotermitídeos, são xilófagos e vivem no interior de troncos secos (Constantino 1992; Eggleton 2000) ou, ocasionalmente, em troncos ainda vivos (Eggleton 2000). Este remanescente florestal localizado às margens de um meandro antigo de rio, tem seu solo recoberto por uma lâmina d'água durante diversos períodos ao longo do ano. Porém, após o recuo das águas, fica totalmente ressecado e compactado, mas que pela quantidade de troncos secos existente favorece a manutenção dos xilófagos como Rugitermes sp. 1 .

A maioria das espécies apresentou abundância intermediária na maior parte dos ambientes. No entanto, Grigiotermes sp.1 e o calotermitídeo não identificado foram raros no remanescente da Área de Preservação, G. bequaerti e Neocapritermes sp.1 no Campestre, sendo que este último táxon só foi coletado neste remanescente. Finalmente, o apicotermitíneo não identificado foi raro na área em sucessão vegetal (Imperatriz Leopoldina).

Exceto o remanescente na Base Ecológica Rio Velho que

Tabela III. Riquezas, observada $\left(\mathrm{S}_{\mathrm{obs}}\right)$ e estimada $\left(\mathrm{S}_{\text {est }}\right)$ e desvio padrão (+/- d.p.), das espécies de térmitas em sete remanescentes florestais e em uma área em sucessão vegetal.

\begin{tabular}{lccc}
\hline \multicolumn{1}{c}{ Ambientes } & $\mathrm{S}_{\mathrm{obs}}$ & $\mathrm{S}_{\mathrm{est}}$ & \pm d.p. \\
\hline$\quad$ Remanescente florestal & & & \\
Área de Preservação & 5 & 6,8 & 1,2 \\
Base Ecológica Rio Velho & 2 & 2,0 & 0,0 \\
São José & 2 & 2,9 & 0,9 \\
Cristo Rei & 2 & 2,0 & 0,0 \\
Morro do Espelho & 5 & 6,8 & 1,8 \\
Campestre & 6 & 8,7 & 1,4 \\
$\quad$ Sucessão vegetal & & & \\
Imperatriz Leopoldina & 5 & 5,0 & 0,0 \\
\hline
\end{tabular}

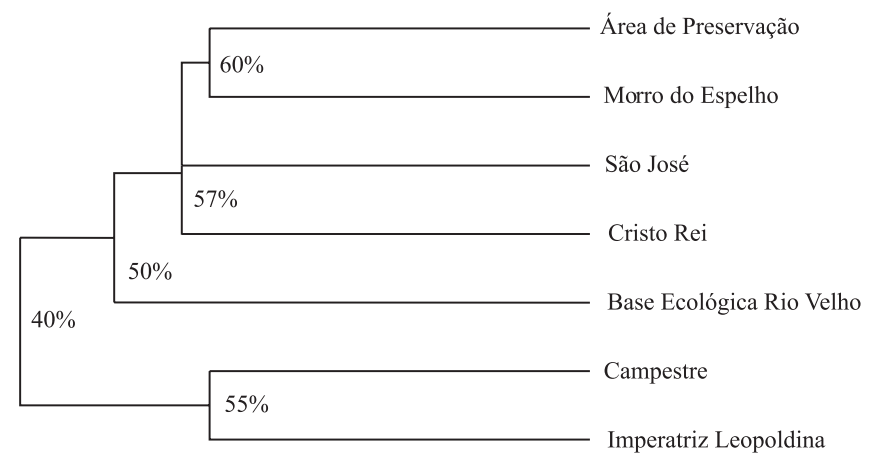

Distância

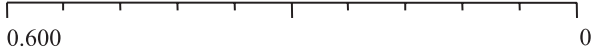

Fig. 2. Análise de agrupamento das comunidades de térmitas dos seis remanescentes florestais e da área em sucessão vegetal.

está sob condições visivelmente estressantes (períodos com solo compactado e ressecado, seguidos por inundações) e que dificultam, especialmente, a sobrevivência de térmitas geófagos, os demais ambientes são atualmente bastante similares. Desta forma, as diferenças observadas na composição e padrão de ocorrência das espécies parece não estar correlacionada com as características ambientais.

A distribuição e a abundância dos organismos estão relacionadas com o clima, com a disponibilidade local de recursos para alimentação e nidificação, bem como são dependentes das relações intra- e interespecíficas existentes e do nível de distúrbios ambientais. A riqueza, abundância e a composição também podem estar relacionadas com outros fatores, tais como estrutura vegetacional (Bandeira \& Macambira 1988), tipos físico-químicos da matéria orgânica do solo (Bignell \& Eggleton 2000) e com efeitos estocásticos como deriva genética, fluxo gênico (migração) e efeito do fundador. Os dados obtidos nos diversos remanescentes avaliados não permitem estabelecer relações entre a termitofauna encontrada e as características locais atuais. $\mathrm{O}$ número de espécies não apresentou correlação com o tamanho da área, o que em parte poderia ser um artefato decorrente do baixo número de setores amostrados e da falta de repetições (Roisin \& Leponce 2004).

Alterações em um ambiente podem favorecer alguns grupos tróficos (Bignell \& Eggleton 2000), ou certas espécies (Wood \& Johnson 1986; Donovan et al. 2002) e levar outras à extinção local (De Souza \& Brown 1994) seja por atuarem diretamente sobre as populações ou por alterarem os habitats (De Souza et al. 2001). Por exemplo, a área em sucessão vegetal (Imperatriz Leopoldina), após seu desmatamento foi aterrada inúmeras vezes, de modo que colônias de térmitas provenientes de outros locais podem ter sido transportadas juntas, favorecendo a emigração (Fontes 1998b; Diehl et al. 2001) e, por consequiência, elevando-se a riqueza de espécies.

A baixa similaridade entre as comunidades de térmitas dos remanescentes florestais e da área em sucessão vegetal, sugere que, originalmente, a floresta estacional semidecidual na região 
apresentava riqueza de espécies não muito mais elevada, o que é típico de zonas de clima subtropical (Eggleton et al.1994) e, possivelmente, uma distribuição heterogênea das espécies, tal como relatado por De Souza \& Brown (1994). Após a fragmentação, os remanescentes mantiveram a baixa riqueza, mas passaram a diferir na composição de espécies. Também o histórico de alterações, o nível de umidade do solo e a estrutura da vegetação de cada ambiente poderiam ter favorecido a formação das distintas comunidades. Segundo De Souza (1995), os remanescentes florestais são importantes porque servem como refúgio para algumas espécies de térmitas que podem estar sendo afetadas pelo crescimento das populações humanas, expansão urbana e agro-industrial. Paralelamente, pela fragmentação das florestas, certas espécies poderiam perder seus predadores e competidores de modo que, na ausência destes constritores, podem se fixar e, inclusive, apresentar um grande crescimento de suas populações. Também com a perda das condições ideais de sobrevivência e reprodução, outras espécies de térmitas poderiam se extinguir ou entrar em processo de extinção local. Essas alterações, em conjunto ou isoladas, aliadas às condições ambientais locais decorrentes do clima subtropical poderiam ser responsáveis pelas diferenças na composição de espécies nos remanescentes da floresta estacional semidecidual e da área em sucessão vegetal avaliados. Finalmente, deve ser considerado que se as populações das diversas espécies não apresentarem um padrão de distribuição homogêneo, isto é, se forem por exemplo agregadas, o baixo número de amostragens e a inadequada área amostrada, aliados a falta de repetições poderão subestimar a riqueza de espécies (De Souza et al. 2001; Roisin \& Leponce 2004). Assim, este trabalho deve ser considerado apenas como um ponto de partida aos estudos sobre a termitofauna no sul do Brasil, sendo que as questões surgidas deverão ser investigadas em trabalhos futuros.

Agradecimentos. Ao Dr. Reginaldo Constantino da UnB pela identificação dos térmitas. À UNISINOS pela Bolsa de Mestrado a primeira autora. À equipe do Laboratório de Insetos Sociais pela ajuda em campo.

\section{REFERÊNCIAS}

Araujo, R. L. 1977. Catálogo dos Isoptera do Novo Mundo. Rio de Janeiro, Academia Brasileira de Ciências, 92 p.

Bandeira, A. G. \& M. L. J. Macambira. 1988. Térmitas de Carajás, estado do Pará, Brasil: composição faunística, distribuição e hábito alimentar. Boletim Museu Paraense Emílio Goeldi, série Zoologia 4: 175-190.

Bandeira, A. G. \& A. Vasconcellos. 2002. A quantitative survey of termites in a gradient of disturbed highland forest in northeastern Brazil (Isoptera). Sociobiology 39: 429-439.

Bignell, D. E. \& P. Eggleton. 2000. Termites in ecosystems, p. 363387. In: T. Abe; D. E. Bignell \& M. Higashi (eds.). Termites: evolution, sociality, symbioses, ecology. Netherlands, Kluwer Academic Publishers, $466 \mathrm{p}$.

Brauman, A.; D. E. Bignell \& I. Tayasu. 2000. Soil-feeding termites: biology, microbial associations and digestive mechanisms, p. 233259. In: T. Abe; D. E. Bignell \& M. Higashi (eds.). Termites: evolution, sociality, symbioses, ecology. Netherlands, Kluwer Academic Publishers, 466 p.
Cancello, E. M. \& T. Schlemmermeyer. 1999. Isoptera, p. 82-91. In: C. R. F. Brandão \& E. M. Cancello (orgs.). Biodiversidade do Estado de São Paulo, Brasil: Síntese do conhecimento ao final do século XX. Invertebrados Terrestres. São Paulo, FAPESP, Vol. 5, 279 p.

Castro, Z. S. C. \& E. Diehl. 2003. Gêneros de térmitas em ninhos epígeos no campus da UNISINOS, São Leopoldo, RS. Acta Biologica Leopoldensia 25: 93-102.

Colwell, R. K. 2004. Estimates: statistical estimation of species richness and shared species from sample. Version 7. [Online] Disponível da WWW: <URL http://purl.ocic.org/estimates>.

Constantino, R. 1992. Abundance and diversity of termites (Insecta: Isoptera) in two sites of primary rain forest in Brazilian Amazonia. Biotropica 24: 420-430.

Constantino, R. 1998. Catalog of the living termites of the new world (Insecta: Isoptera). Arquivos de Zoologia 35: 135-260.

Constantino, R.1999. Chave ilustrada para identificação dos gêneros de cupins (Insecta: Isoptera) que ocorrem no Brasil. Papéis Avulsos de Zoologia 40: 387-448.

Davies, R. G. 1997. Termite species richness in fire-prone and fireprotected dry deciduous dipterocarp forest in Doi Suthep-Pui National Park, northern Thailand. Journal of Tropical Ecology 13: $153-160$.

Davies, R. G. 2002. Feeding group responses of a neotropical termite assemblage to rain forest fragmentation. Oecologia 133: $233-$ 242.

De Souza, O. 1995. Efeitos da fragmentação de ecossistemas em comunidades de cupins, p. 19-27. In: E. Berti Filho \& L. R. Fontes (eds.). Biologia e controle de cupins. Piracicaba, FEALQ, $183 \mathrm{p}$.

De Souza, O. F. F. \& V. K. Brown. 1994. Effects of habitat fragmentation on Amazonian termite communities. Journal of Tropical Ecology 10: $197-206$.

De Souza, O.; J. H. Schoereder; V. Brown \& R. O. Bierregaard Jr. 2001. A theoretical overview of the process determining species richness in forest fragments, p. 13-21. In: R. O. Bierregaard Jr.; C. Gascon; T. E. Lovejoy \& R. C. G. Mesquita (eds.). Lessons from Amazonia: the ecology and conservation of a fragmented forest. New Haven, USA, Yale University Press, 478 p.

Diehl, E.; A. M. de Araújo \& S. Cavalli-Molina. 2001. Genetic variability and social structure of colonies in Acromyrmex heyeri and A. striatus (Hymenoptera: Formicidae). Brazilian Journal of Biology 61 : 667-678.

Diehl, E.; D. F. Florencio; F. A. Schmidt \& L. V. A. Menzel. 2005. Riqueza e composição das comunidades de formigas e térmitas na Floresta Nacional de São Francisco de Paula (FLONA-SFP), RS. Acta Biologica Leopoldensia 27: 99-106.

Diehl-Fleig, E.; M. E. Da Silva \& R. Castilhos-Fortes. 1995. O problema dos cupins no Rio Grande do Sul, p. 53-56. In: E. Berti Filho \& L. R. Fontes (eds.). Alguns aspectos atuais da biologia e controle de cupins. Piracicaba, FEALQ, $184 \mathrm{p}$.

Donovan, S. E.; P. Eggleton \& A. Martin. 2002. Species composition of termites of the Nyika plateau forests, northern Malwi, over an altitudinal gradient. African Journal of Ecology 40: 379-385.

Eggleton, P. 1994. Termites live in a pear-shaped world: a response to Platnick. Journal of Natural History 28: 1209-1212.

Eggleton, P.; P. M. Williams \& K. J. Gaston 1994. Explaining global termite diversity: productivity or history? Biodiversity and Conservation 3: 318-330.

Eggleton, P. 2000. Global patterns of termite diversity, p. 25-51. In: T. Abe; D. E. Bignell \& M. Higashi (eds.). Termites: evolution, sociality, symbioses, ecology. Netherlands, Kluwer Academic Publishers, $466 \mathrm{p}$.

Eggleton, P.; D. E. Bignell; W. A. Sands; B. Waite; T. G. Wood \& J. H. Lawton. 1995. The species richness of termites (Isoptera) under differing of forest disturbance in the Mbalmayo Forest Reserve, southern Cameroon. Journal of Tropical Ecology 11: 85-98.

Fontes, L. R. 1985. Potentialities of the appearance of the worker gut in situ for the identification of neotropical genera of Apicotermitinae (Isoptera, Termitinae). Annals of Entomology 3: $1-6$. 
Fontes, L. R. 1992. Key to the genera of the New World Apicotermitinae (Isoptera: Termitinae), p. 242-248. In: D. Quintero \& A. Aiello (eds.). Insects of Panama and Mesoamerica. Selected studies. New York, Oxford University Press, 692 p.

Fontes, L. R. 1998a. Novos aditamentos ao "Catálogo dos Isoptera do Novo Mundo", e uma filogenia para os gêneros neotropicais de Nasutitermitinae, p. 309-412. In: L. R. Fontes \& E. Berti Filho (eds.). Cupins. O desafio do conhecimento. Piracicaba, FEALQ, $512 \mathrm{p}$.

Fontes, L. R. 1998b. Considerações sobre a complexidade da interação entre o cupim subterrâneo, Coptotermes havilandi, e a arborização no ambiente urbano, p. 109-124. In: L. R. Fontes \& E. Berti Filho (eds.). Cupins. O desafio do conhecimento. Piracicaba, FEALQ, $512 \mathrm{p}$.

Fontes, L. R. \& R. L. de Araujo. 1999. Os cupins, p. 35-90. In: F. A. M. Mariconi (coord.). Insetos e outros invasores de residências. Piracicaba, FEALQ, 460 p.

Hackbart, E. J. 2004. Serviço de Meteorologia da Secretaria Municipal de Meio Ambiente (SEMMAM). São Leopoldo. Referência obtida na Internet. <http://www.semmamsaoleo.kit.net> (acessado em 27 de setembro de 2004).

Jones, D. T. \& P. Eggleton. 2000. Sampling termite assemblages in tropical forests: testing a rapid biodiversity assessment protocol. Journal of Applied Ecology 37: 191-203.

Kambhampati, S. \& P. Eggleton. 2000. Taxonomy and phylogeny of termites, p. 1-23. In: T. Abe; D. E Bignell \& M. Higashi (eds.). Termites: evolution, sociality, symbioses, ecology. Netherlands, Kluwer Academic Publishers, 466 p.
Krebs, C. J. 1997. Ecological Methodology. Version 0.9. [Online] Disponível da WWW: <URL http://gause/biology/ualberta.ca/pub/ jbrzusto/krebs>.

Mathews, A. G. A. 1977. Studies on termites from the Mato Grosso State, Brazil. Rio de Janeiro, Academia Brasileira de Ciências, 267 p.

Mill, A. E. 1982. Populações de térmitas (Insecta, Isoptera) em quatro habitats no baixo Rio Negro. Acta Amazonica 12: 53-60.

Roisin, Y. \& M. Leponce. 2004. Characterizing termite assemblages in fragmented forests: A test case in the Argentinian Chaco. Austral Ecology 29: 637-646.

Scatolini, D. \& A. M. Penteado-Dias. 2003. Análise faunística de Braconidae (Hymenoptera) em três áreas de mata nativa do Estado do Paraná, Brasil. Revista Brasileira de Entomologia 47: 187195.

Teixeira, M. B.; A. B. Coura Neto; U. Pastore \& A. L. R. Rangel Filho. 1986. Vegetação. As regiões fitoecológicas, sua natureza e seus recursos econômicos. Estudo fitogeográfico, p. 541-632. In: Levantamento de recursos naturais. Rio de Janeiro, Instituto Brasileiro de Geografia e Estatística, 791 p.

Wilkinson, L. 2000. SYSTAT: the system for statistics. Evanston, Illinois.

Wood, T. G. 1975. The effects of clearing and grazing on the termite fauna (Isoptera) of Tropical savannas and woodlands, p. 409418. In: J. Vanek. Progress in Soil Zoology. Prague, Academia, $630 \mathrm{p}$.

Wood, T. G. \& R. A. Johnson. 1986. The biology, physiology, and ecology of termites, p. 1-68. In: S. B. Vinson (ed.). Economic impact and control of social insects. New York, Praeger, $421 \mathrm{p}$. 